\title{
Caracterización de tipologías de productores de plátano (Musa AAB) en los Llanos Orientales de Colombia
}

\section{Characterization of plantain (Musa AAB) farmers typologies in the Llanos Orientales of Colombia}

\author{
Gustavo A. Rodríguez-Yzquierdo*๑; Julio J. Becerra-Campiño ${ }^{2 \oplus}$; Tatiana C. Miranda-Salas ${ }^{2}$; \\ Sandra V. Alzate-Henao ${ }^{3 \odot}$; Hector A. Sandoval-Contreras ${ }^{4 \odot}$ \\ Recibido para publicación: Febrero 28 de 2019 - Aceptado para publicación: Mayo 24 de 2019

\section{RESUMEN} \\ ABSTRACT
}

Los Llanos Orientales ha sido tradicionalmente una de las regiones más productivas del cultivo de plátano. Sin embargo, la clasificación convencionalmente aplicada a los productores ha sido, como pequeño (1-5 ha), mediano (6-15 ha) y grande (>15 ha), sólo en función al área de siembra establecida, sin considerar ningún criterio técnico ni económico que permita identificar otros factores que logren diferenciar a los platanicultores de esta zona del país. Considerando esto, se realizó una caracterización técnica, económica y social de los tipos de productores de plátano en los departamentos de Meta, Casanare y Arauca con base a la información colectada a través de una encuesta aplicada. Los municipios seleccionados fueron Granada y Fuentedeoro en el Meta, Yopal y Pore en Casanare, Tame y Saravena en Arauca. La encuesta abordó los aspectos técnicos del cultivo, permitiendo además obtener información de línea base que permitió el análisis del estado actual en cuanto adopción tecnológica de la región. Con el análisis de los datos obtenidos, se definieron las siguientes tipologías: 1. Productores con alta productividad, alta adopción tecnológica y alta inversión financiera; 2. Productores con mediana productividad, alta adopción tecnológica e inversión financiera intermedia; 3. Productores con mediana a baja productividad, baja adopción tecnológica y baja inversión financiera y 4. Productores con baja productividad, baja adopción tecnológica y baja inversión financiera. Con estos resultados, se definen los puntos críticos en cada tipología y de esta manera, diseñar los planes de trabajo e investigación requeridos para mejorar el sistema productivo para cada una de ellas.

Palabras clave: Adopción tecnológica; Clasificación; Finanzas; Musáceas; Producción.

\footnotetext{
${ }^{1}$ Red de Frutales Agrosavia Sede Central Mosquera, Cundinamarca, Colombia.

${ }^{2}$ Red de Frutales Agrosavia Cl La Libertad Villavicencio, Meta, Colombia.

${ }^{3}$ Red de Frutales Agrosavia CI La Selva Sede Manizales, Caldas, Colombia.

${ }^{4}$ Red de Frutales Agrosavia Cl La Libertad Sede Yopal, Casanare, Colombia.

*Autor para correspondecia: Gustavo Rodríguez

Email: grodriguezy@agrosavia.co
}

The Llanos Orientales has traditionally been one of the most productive plantain regions. However, farmer's classification has always been as small (1-5 ha), medium (6-15 ha) and big (>15 ha) according to the planting area, without considering any other technical or economic criteria that would allow to differentiate plantain farmers in this area of the country. A economic, technical and social characterization of plantain producers in the departments of Meta, Casanare and Arauca was done based on a survey in the municipalities of Granada and Fuentedeoro in Meta, Yopal and Pore in Casanare, Tame and Saravena in Arauca. The survey addressed all crop technical factors from soil preparation to post-harvest of plantain. Data analysis resulted in the following farmer's typologies: 1. Producers with high productivity, technology and financial investment; 2 . Producers with medium productivity, high technology and intermediate financial investment; 3. Producers with medium to low productivity, low technology and low financial investment and 4. Producers with low productivity, low technology and low financial investment. The results defined critical points for each typology and the facts to design work and research plans required to improve the production system for each type.

Key words: Technological adoption; Classification; Finance; Musaceae; Production.

\section{Cómo Citar}

Rodríguez-Yzquierdo, G., Becerra-Campiño, J., MirandaSalas, T., Alzate-Henao, S. y Sandoval-Contreras, H. 2019. Caracterización de tipologías de productores de plátano (Musa $\mathrm{AAB})$ en los Llanos Orientales de Colombia. Temas Agrarios 24(2): 129-138. https://doi.org/10.21897/rta.v24i2.2001

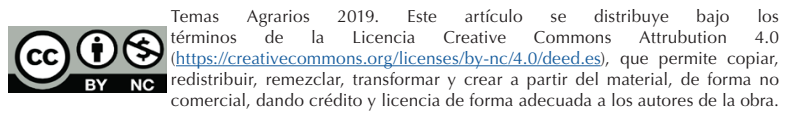




\section{INTRODUCCIÓN}

El cultivo de plátano en Colombia tuvo una producción 4.316.727 t durante el 2017, de los cuales los departamentos de Arauca, Meta y Casanare-región Llanos Orientales participaron con un $22,78 \%$ de la producción total, es decir, con 961.694 t cosechadas en 50.436 ha, con un rendimiento de 19,07 toneladas/hectárea (t.ha- ${ }^{1}$ ) (Ministerio de Agricultura y Desarrollo Rural, Departamento Administrativo Nacional de Estadística y Secretarías municipales, 2017).

Tradicionalmente, los productores de plátano a nivel nacional han sido clasificados precisamente de acuerdo con el área establecida de este cultivo, como pequeños (entre 0,1-5 ha), medianos (5,1-15 ha) y grandes (15,1-30 ha) (Ministerio de Agricultura y Desarrollo Rural, 2014) y aquellos con más de 30 ha son considerados como empresariales (Rodríguez y Rodríguez, 1999; Roldán et al., 2002; Espinal et al., 2005). En Urabá, por ejemplo, un $90 \%$ del plátano que se produce es de empresas familiares y fincas de pequeños productores, mientras que en los Llanos Orientales esta proporción es del 73\% (Espinal et al., 2005).

Para el caso de los Llanos Orientales, se han adelantado algunos estudios que han permitido establecer ese porcentaje de productores de acuerdo con dichas categorías. Uno de ellos, realizado por Rojas (2018), encontró que en Arauquita (Arauca) de las fincas cultivadas en plátano, 56,66\% pertenecen a pequeños productores con áreas entre $1-5$ ha; los medianos (30\%) tienen áreas entre 6-14 ha; $13,33 \%$ corresponde a grandes con 15-30 ha.

Sin embargo, a pesar de que estas categorías de clasificación para los platanicultores han sido utilizadas de forma transversal en diferentes escenarios del sector agropecuario como la investigación, planificación de proyectos, créditos, entre otros; no considera ningún criterio técnico diferente al número de hectáreas sembradas, que permitan categorizar a los productores en formas funcionales y de esta forma poder establecer análisis más profundos y diferenciados, con miras a desarrollar estrategias más precisas de intervención en el sistema productivo.

Por ejemplo, en Nicaragua donde a pesar de que los productores de plátano se clasifican también por el tamaño del área establecida, pequeños (0-3,5 ha), medianos (3,5-35 ha) y grandes (>70 ha), adicionalmente, evalúan algunas variables técnicas como variedad cultivada, presencia de enfermedades, rendimiento y manejo del material de siembra (Instituto Interamericano de Cooperación para la Agricultura, Japan International Cooperation Agency y Ministerios Agropecuario y Forestal, 2004).

Otro caso similar de tipificación, pero para los pequeños productores de plátano está en el municipio de Obispo, estado Barinas, Venezuela, donde se encontró que existen seis tipos de productores dependiente de la edad, procedencia, vivienda, condiciones sanitarias, alumbrado, casa parcela, aplicación de abono, mano de obra, peso del fruto, largo del fruto, como vende el producto, asistencia técnica y capacitación (Delgado et al., 2004). En esta investigación se consideran otros elementos adicionales de carácter sociocultural, técnicos y económicos.

Por su parte, en la región de los Llanos Orientales, se encontró que en el Meta los productores de plátano manejan el sistema productivo de la siguiente manera (Ecopetrol y País Rural, 2014): Sistema Tradicional de producción: densidad siembra, 750 a 1100 plántulas/ha; costos establecimiento, $\$ 8.074 .000$ por ha; costos sostenimiento, $\$ 3.755 .000$ por ha, con un rendimiento producción 9 a 12 t.ha- ${ }^{1}$ año; Sistema Tecnificado con riego: densidad de siembra, 2000 a 2500 plántulas/ha; Costos de Establecimiento, \$18.930.000 por ha; Costos de Sostenimiento, \$7.843.000, con rendimientos de producción de 22 a 29 t.ha- ${ }^{1}$ año; y, Sistema Tecnificado sin riego: densidad de siembra, 2000 a 2500 plántulas/ha; costos de establecimiento, 
\$11.480.000 por ha; costos de sostenimiento, $\$ 7.843 .000$, con rendimientos de producción 18 a 20 t.ha- $^{1}$ año. Sin embargo, este estudio no clasifica a los productores, sino al manejo de sistema productivo de forma general.

A través de la investigación agrícola se logra el aporte de conocimientos para mejorar la rentabilidad de los cultivos y conservar los recursos naturales (Damián et al., 2007). Por esto, los investigadores de Agrosavia, Cl La Libertad realizaron este trabajo de investigación con la finalidad de desarrollar una tipificación funcional de sistemas productivos de plátano en los departamentos de Arauca, Meta y Casanare, teniendo en cuenta su productividad, adopción tecnológica e inversión para el manejo del cultivo de plátano. Mediante esta tipificación se busca agrupar a los productores con características de manejo, producción y técnicas similares (Hart, 1990; Berdegué y Larrain, 1988; Malagón y Prager, 2001; Álvarez et al., 2019), según su lógica económica, que es la expresión del tipo de recursos que posee, de su habilidad y experiencia tecnológica y de las limitantes y potencialidades de la zona (Food and Agriculture Organization of the United Nations y Universidad San Carlos de Guatemala, 1995).

De esta manera, al caracterizar a los agricultores de este sistema productivo, se reconoce cuáles son factores que prevalecen en cada uno de ellos (Arias et al., 1990) lo cual permita establecer las estrategias de manejo diferenciadas que orienten a la mejora del sistema productivo con base a recomendaciones de manejo y posibles líneas de investigación estratégicas orientadas al entorno y condiciones específicas de los tipos de productores que existen en la región de los Llanos Orientales.

\section{MATERIALES Y MÉTODOS}

El trabajo de investigación se realizó en los departamentos de Meta, Casanare y Arauca.
En cada departamento se seleccionaron los dos municipios con mayor producción de plátano, los cuales fueron: Granada y Fuente de Oro (Meta); Aguazul y Pore (Casanare); Tame, y Saravena (Arauca). Para tal fin, se diseñó y aplicó una encuesta diagnóstica, a través de la cual se obtuvo información técnica, productiva y económica, con un tamaño de muestra de 98 productores escogidos bajo los criterios de experiencia, tradición y conocimiento del cultivo.

La encuesta tuvo un total de 226 variables distribuidas en 11 componentes que fueron: información del productor, información del predio, información del cultivo, semilla, siembra, labores culturales realizadas, fertilización, control de plagas, control de enfermedades, cosecha y asistencia técnica en el cultivo de plátano.

La aplicación de las encuestas se realizó bajo dos técnicas de recolección de información: grupal y personalizada. La primera, se desarrolló con grupos de productores miembros de asociaciones; la segunda, se realizó directamente en predios con cada productor, y en ambas, el diligenciamiento estuvo bajo la dirección de personal Agrosavia, CI La Libertad.

Después de recopilar la información, se realizó la tabulación, procesamiento, análisis e interpretación de resultados. La categorización y selección de variables se realizó mediante un análisis exploratorio (distribución de frecuencias), para el total de las variables contempladas, de allí se escogieron aquellas que permitieran definir tipologías de productores de acuerdo con el nivel de adopción tecnológica.

Posteriormente, se procedió con la caracterización de tipologías de productores de plátano mediante un análisis de correspondencia múltiple (ACM) (Der y Everitt, 2001), a través del cual se identificaron las variables, dimensiones y categorías que describían al sistema productivo. Finalmente, se realizó un 
análisis de conglomerados (Der y Everitt, 2001), el cual permitió agrupar los agricultores a partir de las variables con respuestas homogéneas en tipologías de productores diferenciados. Estos análisis multivariados fueron desarrollados a través del software estadístico $\mathrm{R}$ versión 3.4.3.

Con la información obtenida de las 98 encuestas, se depuraron los datos y se conformó una matriz con 191 variables cualitativas, de las cuales, a través del análisis de correspondencia múltiple, se realizó la selección de 10 variables teniendo en cuenta su poder discriminante para lograr la caracterización y tipificación, ya que representaban un $62,06 \%$ de la expresión de la variabilidad del total de variables estudiadas, lo cual es adecuado de acuerdo con Pla (1986) e lezzoni y Pritts (1991), quienes señalan que valores superiores al $40 \%$ suelen ser suficientes para realizar análisis adecuados. Esas variables fueron: área (ha), densidad de siembra (plantas/ ha), fertilización, riego, control químico de plagas y enfermedades, control biológico de plagas, rendimiento (t.ha- ${ }^{1}$ ), costo promedio de la producción (pesos/ha) y asistencia técnica.

\section{RESULTADOS Y DISCUSIÓN}

Los resultados obtenidos a partir el ACM para las 10 variables muestran una baja contribución de cada dimensión a la variabilidad total, expresada en bajos porcentajes de varianza explicada. Por tal razón, se seleccionaron 7 dimensiones o factores que conjuntamente explican el $62 \%$ de la inercia total (Tabla 1 ). Cada variable estuvo a su vez representada por una serie de categorías y dentro de estas últimas, las que mayormente aportaron al porcentaje de variación acumulado fueron: áreas mayores a 10 ha; densidades de siembra de 1600 plantas/ ha, 2000 plantas/ha y no tradicionales; ausencia de fertilización; control biológico de plagas; bajos rendimientos $\left(<15\right.$ t.ha- $\left.{ }^{1}\right)$, rendimientos intermedios (15-35 t.ha- $\left.{ }^{1}\right)$ y altos rendimientos (>35 t.ha- ${ }^{1}$ ); bajos, intermedios y altos costos de producción, que oscilaron entre valores menores a 7 millones de pesos y mayores a 15 millones.
Tabla 1. Análisis de correspondencia múltiple de los datos de la encuesta a productores de plátano de los Llanos Orientales. 2017, Colombia.

\begin{tabular}{|c|c|c|c|}
\hline Dimensiones & Varianza & $\begin{array}{c}\text { Porcentaje } \\
\text { Varianza }\end{array}$ & $\begin{array}{c}\text { Porcentaje } \\
\text { acumulado } \\
\text { Varianza }\end{array}$ \\
\hline 1 & 0,26 & 14,57 & 14,57 \\
\hline 2 & 0,18 & 10,13 & 24,70 \\
\hline 3 & 0,16 & 9,31 & 34,01 \\
\hline 4 & 0,14 & 8,08 & 42,09 \\
\hline 5 & 0,12 & 7,11 & 49,21 \\
\hline 6 & 0,11 & 6,61 & 55,82 \\
\hline 7 & 0,11 & 6,24 & 62,06 \\
\hline 8 & 0,10 & 5,56 & 67,62 \\
\hline 9 & 0,09 & 5,14 & 72,76 \\
\hline 10 & 0,08 & 4,75 & 77,51 \\
\hline 11 & 0,07 & 4,29 & 81,80 \\
\hline 12 & 0,07 & 4,23 & 86,03 \\
\hline 13 & 0,05 & 3,12 & 89,15 \\
\hline 14 & 0,05 & 3,03 & 92,18 \\
\hline 15 & 0,05 & 2,91 & 95,09 \\
\hline 16 & 0,04 & 2,49 & 97,58 \\
\hline 17 & 0,02 & 1,59 & 99,18 \\
\hline 18 & 0,01 & 0,82 & 100 \\
\hline
\end{tabular}

Fuente: Los autores

A partir de estos resultados, se realizó el análisis de conglomerados (Der y Everitt, 2001) que minimiza las varianzas entre los grupos y maximiza las varianzas dentro de cada grupo. Mediante la aplicación de este análisis se pudo determinar cuatro grupos o tipologías de productores de plátano en los Llanos Orientales, con base a la población muestreada. La figura 1 muestra la agrupación en clúster de los 4 grupos de productores diferenciados por las variables que se definen a continuación: 


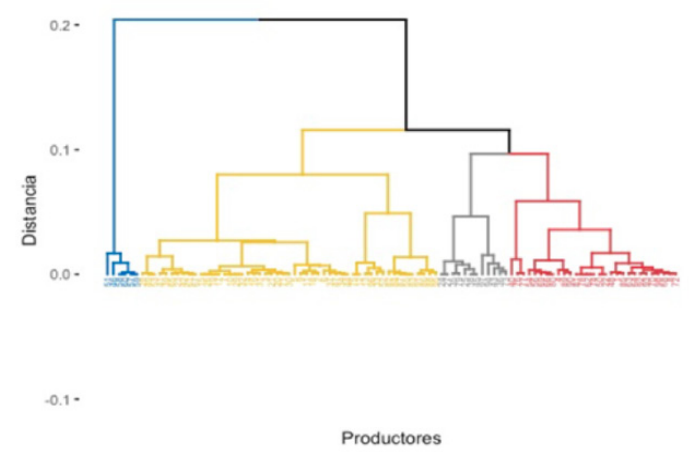

Figura 1. Dendograma de 4 tipos de productores del cultivo de plátano en la región de los Llanos Orientales. Fuente: Los autores

Grupo 1: Conformado por el $53 \%$ de los productores. En su mayoría este grupo se caracteriza por tener áreas cultivadas mayores a 10 hectáreas, con acompañamiento técnico. Utilizan altas densidades de siembra (2.500 plantas/ha) a un solo ciclo de producción, con rendimientos entre 25 y 30 t.ha- $^{1}$ y una inversión entre los 11 y 15 millones de pesos. Este grupo presenta un esquema de prácticas de cultivo tendientes a optimizar la producción, ejecutan planes de fertilización, aplican control biológico para protección del cultivo e implementan sistema de riego.

Grupo 2: Representa el $13 \%$ de los productores encuestados. Se diferencian por presentar densidades de siembra más bajas (entre 2.000 a 2.500 plantas/ha), áreas mayores a 10 ha, con inversiones mayores a los 15 millones de pesos y rendimientos superiores a las 35 t.ha- $^{1}$. Realizan manejo integrado de plagas y enfermedades a través de prácticas culturales, uso de productos químicos e implementan control biológico, hacen aplicación de riego y cuentan con asistencia técnica para la toma de decisiones en sus predios.

Grupo 3: Comprende el 27\% de la población muestral, caracterizado por poseer áreas entre 5 a 10 hectáreas de plátano, sin acompañamiento técnico. Utilizan diferentes densidades de siembra en un rango entre 816 a 2.222 plantas/ha. No implementan sistema de riego y las prácticas de protección del cultivo están orientadas al control químico. Los costos de producción oscilan entre los 7 y 11 millones de pesos con rendimientos de 15 a 25 t.ha- ${ }^{1}$.

Grupo 4: Agrupa el 7\% de los productores, los cuales se distinguen por tener áreas menores a 5 ha, no fertilizan, no cuentan con asistencia técnica, realizan una inversión menor a los 7 millones de pesos y obtienen una producción menor a las 15 t.ha- ${ }^{1}$.

La definición de estos grupos permite diferenciar a los productores de los Llanos Orientales con otras partes del país, por ejemplo, Ruíz y Mejía (2014) y Mejía, Molina, Restrepo y Ruíz (2017), determinaron que en el departamento del Quindío el 52,5\% de los productores tenían entre 1 y 3 ha de plátano y para el caso de los Llanos Orientales, los resultados de investigación arrojaron que solo el $7 \%$ de los productores tienen áreas menores a las 5 ha.

De esta manera, al interpretar los grupos de productores encontrados y las características o variables que los definen, se proponen cuatro tipologías de productores en los Llanos Orientales (Arauca, Casanare y Meta) con base al tipo de manejo del sistema productivo, su productividad y adopción tecnológica, así como el uso de recursos financieros. En cuanto a productividad se definió como baja, mediana y alta de acuerdo con el volumen de producción cosechado y el valor de promedio de referencia de la región que es de 19,07 t.ha- ${ }^{1}$ (MADR et al., 2017).

De este modo, se plantea como alta producción aquella con valores $>30$ t.ha- ${ }^{1}$, mediana entre 19 y 30 t/ha y baja $<19$ t.ha- ${ }^{1}$. Teniendo en cuenta estos criterios, se define entonces las tipologías de la siguiente manera:

Tipología 1: productores con alta productividad, alta adopción tecnológica y alta inversión financiera. Se corresponde con el grupo 2. 
Tipología 2: productores con mediana productividad, alta adopción tecnológica e inversión financiera intermedia. Se corresponde con el grupo 1.

Tipología 3: productores con mediana a baja productividad, baja adopción tecnológica y baja inversión financiera. Se corresponde con el grupo 3.

Tipología 4: productores con baja productividad, baja adopción tecnológica y baja inversión financiera. Se corresponde con el grupo 4.

Por otra parte, las tipologías definidas estuvieron relacionadas directamente con la ubicación geográfica del productor. Es así como la tipología 1 en su gran mayoría se encuentra representada por productores del departamento del Meta con un 28,5\%; la tipología 2 por productores del departamento de Casanare con $6,1 \%$; la tipología 3 por productores del departamento de Arauca, representada en $21,4 \%$ y la tipología 4 nuevamente por el departamento de Casanare con un porcentaje de participación del 6,12\% (Figura 2).

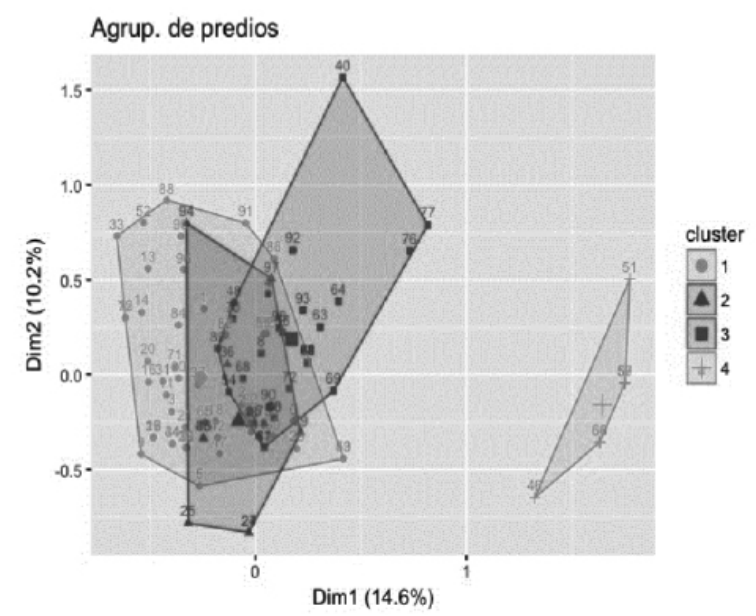

Figura 2. Tipologías definidas de acuerdo con agrupación de productores. Numeración del 1-33: productores del departamento del Meta; 34-60: productores del departamento del Casanare; 61-98: productores del departamento de Arauca. Fuente: Los autores

En este sentido, se puede evidenciar la heterogeneidad de los tipos de sistemas productivos en función a las características particulares de los departamentos en los cuales se desarrolla la actividad productiva. De este modo, la estrategia para implementar esquemas de mejoras desde el punto de vista técnico, pueden variar dependiendo de la particularidad del tipo de agricultor y no solamente considerando el área de siembra de este. Por ello, las recomendaciones de manejo no pueden ser genéricas y deben tener en cuenta su nivel de adopción tecnológica, capacidad de inversión y brecha productiva a la cual se desee cubrir con el impacto de las recomendaciones.

La definición de estas tipologías permite empezar a diferenciar a los productores de plátano de las distintas regiones del país. Por ejemplo, Ruiz y Mejía (2014) encontraron que en el departamento del Quindío, también existen 4 tipologías de productores en el cultivo de plátano: el primer grupo o clúster corresponde al $32,79 \%$ de los productores; el segundo clúster equivale al 49,18\% de los productores; el tercer clúster son el 14,75\% de los productores y, el cuarto clúster equivale al 3,28\% de los productores, Sin embargo, el énfasis de ese estudio en el Quindío responde a las prácticas que realiza el productor orientado a la sostenibilidad del cultivo, desde el ámbito social, productivo y económico, pero no considera de manera particular las variables técnicas del manejo del cultivo, caso contrario al del presente estudio.

Así mismo, otra clasificación que se ha dado a los productores de plátano en el Quindío fue propuesta por Correa (2010), quien tuvo en cuenta las prácticas agropecuarias, el canal de producción al que se accede y la localización de los predios. Lastipologíasfueron: 1) Productores empresariales, 2) Grandes productores tecnificados, 3) Grandes productores extensivos, 4) Pequeños y medianos tecnificados, 5) Pequeños productores no tecnificados y 6) Pequeños productores integrados a Asociaciones y Alianzas. 
De esta forma, con el presente trabajo se aportan características diferenciales robustas, que lo distinguen de clasificaciones normalmente aplicadas a los productores de plátano en función al área de siembra. Esto permite una posible intervención diferenciada en los planes de fomento, actividades de investigación o recomendaciones tecnológicas de las entidades correspondientes en cada caso, para contribuir a la mejora del sistema productivo.

La tipificación de los productores no solo del cultivo de plátano, sino de cualquier sistema productivo permite definir el potencial para mejorar la productividad (Vélez, Espinosa, Amaro y Arechavaleta, 2016). De igual modo, establecer formas de gestión rural bien diferenciadas entre sí, con la detección de debilidades y fortalezas, y establecer prioridades a la hora de diseñar políticas de desarrollo para cada zona (Coronel y Ortuño, 2005; Vélez et al., 2016) y así, incrementar el uso de innovaciones tecnológicas que incidan en una mayor eficiencia y productividad (Cuevas, et al., 2016).

Para lograr esa innovación y adopción de tecnología, es importante que exista la asistencia técnica integral y especializada para cada sistema productivo, de esa manera es posible lograr que la transferencia de tecnología aporte al grado de modernización de la actividad agrícola y de la sociedad rural, tal como propusieron Rogers y Svenning (1979) con su teoría de "difusión de innovaciones" para investigar los factores que afectan dicha adopción, lo cual es esencial para que una sociedad tradicional transite hacia la modernización.

Al organizarse los productores de acuerdo a cada tipología definida en los Llanos Orientales, no solo se favorece la identificación de los puntos críticos a tratar, a su vez también le permite conocer al platanicultor como aumentar su rentabilidad y definir un rumbo dentro de su modelo de negocio, que le brinde una vida mejor para su familia y colegas agricultores, así como una mayor autonomía en la toma de decisiones, conservación de recursos productivos y una relación más equitativa con el mercado (Larqué et al., 2009).

De esta forma, entendiendo la relación entre las tipologías definidas en este estudio para los Llanos Orientales, se pueden diseñar estrategias de acción para cada una de ellas, logrando escalar posiciones hacia sistemas más eficientes agronómica y financieramente (Figura 3).

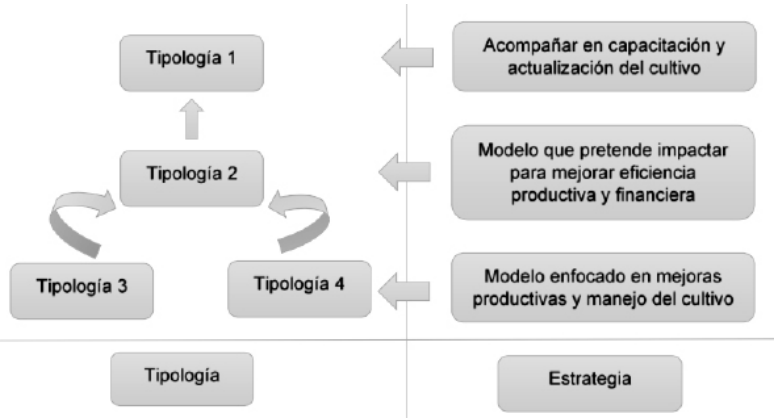

Figura 3. Estrategias para mejorar la tipología del productor de plátano en los Llanos Orientales. Fuente: Los autores.

Con las diferentes estrategias que se implementan de acuerdo con la tipología en la cual se encuentran, es posible lograr un uso eficiente de los recursos en cuanto a insumos agrícolas e inversión financiera, de esta manera los agricultores puedan lograr ser más competitivos y/o rentables. Por otro lado, mejorar las prácticas agronómicas para hacer el sistema más productivo, incrementando el rendimiento por unidad de área. En ambos casos la estrategia debe ser acompañada de entidades con conocimiento del sistema, que aporten los elementos de tecnología e innovación que permitan la mejora sustancial del sistema productivo.

\section{CONCLUSIONES}

Los productores de plátano en la región de los Llanos Orientales se ubican en cuatro 
categorías, de acuerdo con su nivel de productividad, adopción tecnológica e inversión para el manejo del cultivo. Esta agrupación, permitirá establecer estrategias técnicas, sociales y de comercialización del cultivo; así mismo, permitirá identificar cuáles son las necesidades que tienen los productores para mejorar la productividad y especialmente la rentabilidad.

\section{Conflicto de Intereses}

Los autores declaran que es un trabajo orginal y no existío conflicto de intereses de ningún tipo en la elaboración y publicación del manuscrito.

\section{REFERENCIAS}

Álvarez, D., Gómez, E. y Ordóñez, H. 2019. Tipología de fincas productoras de arveja (Pisum sativum L.) en la subregión Sur de Nariño, Colombia. Ciencia Tecnología Agropecuaria, Mosquera (Colombia), Vol. 20 (3): 659-677. ISSN 0122-8706.

Arias, J., Balcázar, A. y Hurtado, R. 1990. Sistemas de producción bovina en Colombia. Coyuntura Agropecuaria Vol. 24: 83-105.

Berdegué, J. y Larraín, B. 1988. ¿Cómo trabajan los campesinos? Serie Producción Agropecuaria Campesina $N^{0}$ 2. Celater, Cali, Colombia.

Coronel, M. y Ortuño, S. 2005. Tipificación de los sistemas productivos agropecuarios en el área de riego de Santiago del Estero, Argentina. Problemas del desarrollo Vol.36 (140): 63-88. México ene/mar. 2005.

Correa, A. 2010. Incidencia del cambio productivo en una sociedad regional: Caso del plátano en el Quindío. Trabajo de grado para optar al título de magíster en Desarrollo Rural. Pontificia Universidad Javeriana. Facultad de Estudios Ambientales y Rurales. Bogotá, Colombia.

https://www.javeriana.edu.co/biblos/ tesis/eambientales/tesis98.pdf
Cuevas, V., Loaiza, A., Espinosa, J., Vélez, A. y Montoya, M. 2016. Tipología de las explotaciones ganaderas de bovinos doble propósito en Sinaloa, México. Revista Mexicana de Ciencias Pecuarias Vol. 7 (1): 69-83 Mérida.

https://doi.org/10.22319/rmcp.v7i1.4150

Damián, M., Ramírez, B., Parra, F., Paredes, J., Gil, A., Cruz, A. y López, J. 2007. Apropiación de tecnología de productores de maíz en el estado de Tlaxcala, México. Agricultura Técnica en México [online]. 2007, Vol. 33 (2), pp.163-173. ISSN 0568-2517.

http://www.scielo.org.mx/ scielo.php?pi d =S0568$\underline{25172007000200006 \& \text { script }=\text { sci arttext }}$

Delgado, E., Paiva, R. y Marín, C. 2004. Caracterización socioeconómica y tipología de los pequeños productores de plátano (Musa AAB subgrupo plátano cv. Hartón Simmonds). Caso: Municipio Obispo, Barinas. Revista de la Facultad de Agronomía (LUZ) Vol 21 (4) Suplemento 1:359-369.

Der, G. y Everitt, B. 2001. A handbook of statistical Analyses using SAS Second Editión. Editorial Chapman y Hall/CRC. ISBN. 1-58488-245 (alk paper). Pag (263284; 307-309).

Ecopetrol y País Rural. 2014. Mejoramiento de la productividad mediante el fomento, transformación y comercialización de plátano con valor agregado para el mercado regional, nacional e internacional en la región de la Orinoquía en el departamento del Meta. Bogotá D. C., marzo 2014. 34 p.

Espinal, C., Martínez, H. y Peña, Y. 2005. Documento de trabajo no. 61. La cadena del plátano en Colombia, una mirada global de su infraestructura y dinámica. http://www.asohofrucol.com.co/ archivos/cadenas/platano.pdf 
Food and Agriculture Organization of the United Nations y Universidad San Carlos de Guatemala. 1995. Caracterización del sistema agrario de la zona de retornados "Nueva Esperanza", Nentón, Huehuetenango. Informe de Proyecto. Guatemala. $66 \mathrm{p}$

Hart, R. 1990. Componentes, subsistemas y propiedades del sistema finca como base para un método de clasificación. En: Escobar G. y Berdegué J. (Eds) Tipificación de sistemas de producción agrícola. RIMISP/GLA. Santiago de Chile. 283 p.

Lezzoni, A. y Pritis, M. 1991. Applications of principal component analysis to horticultural research. HortScience 26(4):334-338.

Instituto Interamericano de Cooperación para la Agricultura, Japan International Cooperation Agency y Ministerios Agropecuario y Forestal. 2004. Cadena agroindustrial plátano. $57 \mathrm{p}$.

http://repiica.iica.int/docs/B0030e/ B0030e.pdf

Larqué, B., Sangerman, D., Ramírez, B., Navarro, A. y Serrano, M. 2009. Aspectos técnicos y caracterización del productor de durazno en el Estado de México, México. Agricultura Técnica en México Vol.35 (3): 305-313. México jul./sep. 2009.

https://bit.ly/2YV3Kc7

MADR Ministerio de Agricultura y Desarrollo Rural; DANE Departamento Administrativo Nacional de Estadística y Secretarías municipales. 2017. Base Agrícola EVA 2007-2017. http://www.agronet.gov.co/Lists/ Boletin/attachments/1322/Base $\% 20$ Agrícola\%20EVA\%202007-2017\%20 (P) $07 \quad 11 \quad 2017 . x I s b$

Ministerio de Agricultura y Desarrollo Rural. 2014. Cadena de plátano. 28 p.
Malagón, R. y Prager, M. 2001. El enfoque de sistemas: Una opción para el análisis de las unidades de producción agrícola. Universidad Nacional de Colombia. Sede Palmira. 190 p.

Mejía, L., Molina, J., Restrepo, L. y Ruíz, J. 2017. Diseño de ecuaciones estructurales para el concepto de sostenibilidad en la agroindustria del plátano en QuindíoColombia. UG Ciencia Revista de Investigación de la Facultad de Ingenierías Vol. 23: 34-45, ISSN 2346-0814.

https://revistas.ugca.edu.co/index.php/ ugciencia/article/view/585

Pla, L. 1986. Análisis multivariado: método de componentes principales. Instituto Interamericano de Estadística. Secretaria General de la OEA. Washington DC. USA. 89 p.

Rodríguez Saavedra, A. y Rodríguez Martínez, J. 1999. Aspectos Socioeconómicos del Cultivo del Plátano en Colombia. Oficina Regional de Planeación - Corpoica, Regional Nueve. Manizales. 29 p.

Rogers, M. y Svenning, L. 1979. La modernización entre los campesinos. Fondo de Cultura Económica. México. $397 \mathrm{p}$

Roldán, D., Salazar, M., Tejada, M., Peña, Y. y Ministerio de Agricultura y Desarrollo Rural. 2002. Documento de trabajo No. 10. Caracterización de la cadena de plátano en Colombia.

http://repiica.iica.int/docs/B0040E/ B0040E.PDF

Rojas, A. 2018. Sistema productivo de plátano Hartón (Musa paradisiaca) para contribuir con el fortalecimiento de la producción agrícola de las comunidades rurales e indígenas de Arauquita (Arauca). Informe final de grado Ingeniería Agronómica. Universidad de la Salle Facultad de Ciencias Agropecuarias. 
Ruiz, M. y Mejía, L. 2014. Condiciones de sostenibilidad del sector productor de plátano del departamento del Quindío bajo el contexto del Reporte de Iniciativa Global (GRI). UG Ciencia Revista de Investigación de la Facultad de Ingenierías Vol. 20 (1): 15-24. ISSN 2346-0814.

https://revistas.ugca.edu.co/index.php/ ugciencia/article/view/309
Vélez, A., Espinosa, J., Amaro, R. y Arechavaleta, M. 2016. Tipología y caracterización de apicultores del estado de Morelos, México. Revista Mexicana de Ciencias Pecuarias. Vol. 7 (4): 507-524.

https://cienciaspecuarias.inifap.gob.mx/ index.php/Pecuarias/article/view/4279 